\title{
Frontières
}

\section{Derrière le miroir de la douleur}

\section{Les interactions médicamenteuses, quiétude ou inquiétude ?}

\section{Andrée Néron}

Volume 17, numéro 2, printemps 2005

Surtout, ne pas souffrir

URI : https://id.erudit.org/iderudit/1073488ar

DOI : https://doi.org/10.7202/1073488ar

Aller au sommaire du numéro

\section{Éditeur(s)}

Université du Québec à Montréal

ISSN

1180-3479 (imprimé)

1916-0976 (numérique)

Découvrir la revue

Citer cet article

Néron, A. (2005). Derrière le miroir de la douleur : les interactions médicamenteuses, quiétude ou inquiétude ? Frontières, 17(2), 42-52. https://doi.org/10.7202/1073488ar
Résumé de l'article

Les statistiques américaines dévoilent que les réactions adverses aux médicaments surviennent en moyenne chez un patient sur 15. Chaque année, environ 2,2 millions de personnes sont touchées par des effets délétères reliés aux médicaments. De ce nombre, les interactions sont imputables dans le tiers des cas et comptent pour la moitié des coûts de santé qui en découlent. On estime que l'incidence d'interactions médicamenteuses est aussi basse que 3 à $5 \%$ pour les personnes consommant peu de médicaments et aussi élevée que 20 à $100 \%$ pour les personnes hospitalisées prenant entre 10 et 20 entités médicamenteuses. Bien que des constellations d'interactions médicamenteuses soient possibles et surviennent, le nombre actuel d'effets adverses manifestes et graves résultant de telles associations est plutôt modeste. Le prescripteur doit savoir anticiper ces interactions en tenant compte des médicaments prescrits, des médicaments en vente libre, des facteurs environnementaux et des co-morbidités.
Ce document est protégé par la loi sur le droit d'auteur. L'utilisation des services d’Érudit (y compris la reproduction) est assujettie à sa politique d'utilisation que vous pouvez consulter en ligne.

https://apropos.erudit.org/fr/usagers/politique-dutilisation/ 


\section{Résumé}

Les statistiques américaines dévoilent que les réactions adverses aux médicaments surviennent en moyenne chez un patient sur 15. Chaque année, environ 2,2 millions de personnes sont touchées par des effets délétères reliés aux médicaments. De ce nombre, les interactions sont imputables dans le tiers des cas et comptent pour la moitié des coûts de santé qui en découlent. On estime que l'incidence d'interactions médicamenteuses est aussi basse que 3 à $5 \%$ pour les personnes consommant peu de médicaments et aussi élevée que 20 à $100 \%$ pour les personnes hospitalisées prenant entre 10 et 20 entités médicamenteuses. Bien que des constellations d'interactions médicamenteuses soient possibles et surviennent, le nombre actuel d'effets adverses manifestes et graves résultant de telles associations est plutôt modeste. Le prescripteur doit savoir anticiper ces interactions en tenant compte des médicaments prescrits, des médicaments en vente libre, des facteurs environnementaux et des co-morbidités.

Mots clés: interactions

médicamenteuses - cytochrome P450

(CYP) - métabolisme -

pharmacocinétique - douleur - soins palliatifs.

\begin{abstract}
American statistics reveal that adverse drug reactions occur in one out of fifteen people taking drugs. It is estimated that approximately 2,2 million people suffer drug related adverse reactions annually. Drug interactions account for a third of drug events and half of the total cost imputed and related to drug injury. Considering all types of drug interactions, it is estimated that as low as $3 \%$ to $5 \%$ of patients taking a small number of drugs to as high as $20 \%$ of those taking 10 to 20 medications may be affected by a drug interaction. Though constellation of drug interactions may happened and supervened, the actual number of serious events are rather modest and manageable. The clinician must anticipate and must use his judgment to evaluate concomitant prescribed drugs, over-thecounter medications, environmental factors and comorbidities.
\end{abstract}

Keywords: drug interactions cytochrome P450 (CYP) - metabolism pharmacokinetics - pain - palliative care.

\section{DERRIÈRE LE MIROIR DE LA DOULEUR Les interactions médicamenteuses, quiétude ou inquiétude?}

\section{Andrée Néron, pharmacienne, D.P.H., Chaire conjointe en douleur, \\ Université du Québec à Trois-Rivières et Université de Sherbrooke.}

Le front plissé, les yeux baissés, la tête préoccupée, vous regardez l'ordonnance édictée; vous constatez que l'être aimé doit prendre au moins cinq comprimés différents pour apaiser sa douleur et stabiliser son hypertension. Vision dantesque ou pharmacothérapie rationnelle à superviser; doit-on s'inquiéter?

Les arcanes de la détoxication du corps humain ont longtemps alimenté notre ignorance et notre impuissance à prédire la réponse d'un individu à une combinaison d'agents pharmacologiques prescrits. Nous affinons notre jugement clinique au fur et à mesure que la science et la phylogenèse nous aident à mieux saisir comment l'organisme s'est doté de mécanismes pour expulser toute forme d'agresseur ingéré ou inhalé et surtout à élucider de quelle façon il parvient à le faire.

La douleur chronique est le plus souvent définie par sa durée qui va au-delà d'une période initiale suivant une lésion ou un traumatisme. La douleur peut être pérennante, épisodique ou un amalgame des deux. La douleur est souvent accompagnée de multiples complaintes satellites incluant un stress émotionnel, une irritabilité, le retrait social, une perte de libido, une détresse financière, des pertur- bations du sommeil, une perte d'appétit et de poids en sus des autres effets pervers de la douleur (nausées et vomissements) et des médicaments employés pour le combat. Les impacts de cette douleur maladie sont tous azimuts aussi faut-il considérer plusieurs éléments dans le plan d'action. L'approche holistique considère toutes les facettes du problème et le plus souvent les professionnels travaillent en multidisciplinarité.

Le régime pharmacothérapeutique pour apaiser la douleur et les autres symptômes connexes doit s'appliquer pour un malade, en fonction de ses caractéristiques biopsychosociales, de la douleur exprimée, de ses expériences antérieures avec la médication. À ce chapitre les notions de pharmacocinétique et de pharmacodynamique sont capitales pour la prise de décision quant à un traitement souvent polypharmaceutique et complexe. Il faut anticiper les interactions potentielles entre les entités prescrites et celles que le patient reçoit déjà pour une autre condition ainsi que les autres entités sans ordonnance et certains aliments que le patient pourrait consommer.

Selon le type ou les types de douleur vécus par le malade, il n'est pas rare de voir prescrire une combinaison de différents agents incluant un antidépresseur, un analgésique non opiacé, un opiacé, un anxiolytique, un anticonvulsivant et un relaxant musculaire pour le même individu (Barkin et al., 2001). 
Une interaction peut s'actualiser lorsque deux ou plusieurs médicaments sont administrés simultanément. Elle se produit lorsqu'un agent modifie l'activité d'une autre substance, en augmentant ou réduisant l'impact clinique pharmacologique (addition, synergie, antagonisme). L'issue de cette interaction peut être bénéfique, si la répercussion est favorable ou nuisible si le résultat s'exprime par une diminution de l'activité ou par des effets délétères pour le malade. Les effets bénéfiques d'une co-administration d'entités synergiques à leur site effecteur peuvent résulter en désagréments secondaires si les doses ne sont pas jugulées. Inversement, l'interaction peut signifier une diminution de l'effet global nécessitant une majoration de la dose de un ou plusieurs médicaments utilisés par le patient (Patsalos et al., 2002).

Les études scientifiques n'ont pas rapporté de données concluantes sur la fréquence ou de l'incidence d'interactions médicamenteuses pour ordonnances prescrites ou sur l'occurrence d'effets adverses imputables à des interactions médicamenteuses dans la population non hospitalisée. Les incidences rapportées pour la clientèle ambulatoire varient entre $9,2 \%$ et $70,3 \%$ pour tous les types d'interactions confondus et de 1,2\% pour les interactions jugées préjudiciables (Bergk et al., 2004).

Les statistiques américaines dévoilent que les réactions adverses aux médicaments surviennent en moyenne chez un patient sur 15 (Davis et Homsi, 2002). Chaque année, environ 2,2 millions de personnes sont touchées par des effets délétères reliés aux médicaments. De ce nombre, les interactions sont imputables dans le tiers des cas et comptent pour la moitié des coûts de santé qui en découlent (Bernard et Bruera, 2000).

Les personnes en phase avancée d'un cancer et recevant des services de soins palliatifs prennent en moyenne cinq entités pharmacologiques ou plus pour pallier les symptômes algiques ou satellites. Ils sont donc à risque d'interactions médicamenteuses.

On estime que l'incidence d'interactions médicamenteuses est aussi basse que 3 à 5\% pour les personnes consommant peu de médicaments et aussi élevée que 20 à 100\% pour les personnes hospitalisées prenant entre 10 et 20 entités médicamenteuses. La difficulté à reconnaître une interaction est reliée au fait qu'il faille distinguer entre la possibilité d'un médicament suspecté, de conditions associées à la maladie ou de facteurs environnementaux (par exemple, le tabagisme) (Bernard et Bruera, 2000).

Dans le contexte de soins palliatifs, plusieurs malades sont âgés. La prévalence de consommation de produits de comptoir (vente libre) chez les personnes âgées affectées par une néoplasie est de $27 \%$. Une autre étude incluant une centaine de malades en unité de soins palliatifs révèle une consommation moyenne de 6,7 médicaments au moment de l'admission et de 10,5 agents pendant leur séjour (Bernard et Bruera, 2000).

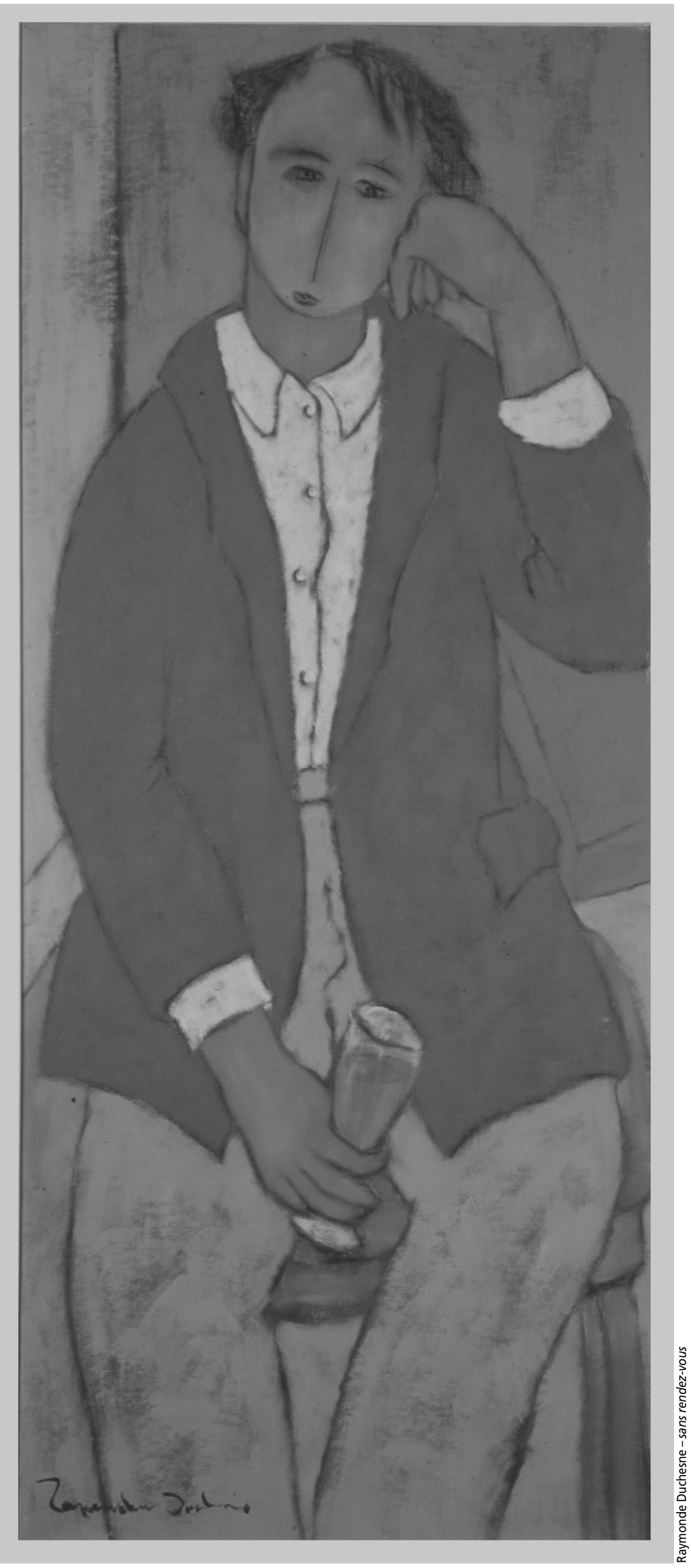


L'administration chez les personnes âgées résulte généralement en pharmacocinétique complexe à cause des changements physiologiques reliés à l'âge et des changements concomitants reliés à la maladie. Les personnes âgées présentent généralement une capacité réduite pour métaboliser les médicaments, une diminution de la liaison aux protéines plasmatiques contemporaine d'une diminution de l'albumine et une diminution de la capacité à éliminer les médicaments par voie rénale (Patsalos et al., 2002).

Bien que des constellations d'interactions médicamenteuses soient possibles et surviennent, le nombre actuel d'effets adverses manifestes et résultant de telles associations est plutôt modeste.

Dans le processus décisionnel qui découle du constat ou de l'anticipation d'une interaction médicamenteuse, le clinicien doit considérer la gravité, le type d'interaction (la façon de composer avec celle-ci), le ratio risque-bénéfice et les facteurs de risque reliés au malade (Huang et al., 2004).

La connaissance des mécanismes d'interaction peut être utilisée à l'avantage du contexte clinique; par exemple, lorsqu'un médicament diminue la vitesse d'élimination et augmente la durée de vie d'un autre médicament visé, la répercussion sur la fréquence d'administration, la diminution de la dose et, conséquemment, l'observance au traitement est favorisée. Chez les malades présentant une concentration sous-thérapeutique du médicament dans le sang, une telle association peut ramener les valeurs à l'intérieur de l'écart pour un meilleur contrôle alors qu'autrement cela ne pourrait pas être possible (sauf au prix de toxicité). Ceci a également et possiblement une valeur en termes de coûts imputables puisque la dose d'agents associés onéreux est conséquemment diminuée (Patsalos et al., 2002).

Les interactions potentielles entre les médicaments peuvent assez souvent être prédites (même si jamais on ne peut être assuré de la réponse individuelle du malade au préalable) grâce à l'information recueillie sur les principes actifs, les connaissances pharmacologiques générales en regard de la classe pharmacologique et à partir des données des phases de recherche précliniques, animales et de l'expérience humaine (Huang et al., 2004).

Pour évaluer le risque qu'une interaction significative se produise et quand celle-ci se produira, il faut tenir compte de plusieurs facteurs:

- histoire médicamenteuse actuelle et récente (le malade pourrait avoir terminé un traitement par médicament ayant des répercussions à long terme même après cessation; p. ex., : fluoxétine $\left[\right.$ Prozac $\left.\left.^{\circledR}\right]\right)$, longue durée de vie de fluoxétine et de son métabolite actif avant l'élimination complète;

VISION DANTESQUE

\section{OU PHARMACOTHÉRAPIE RATIONNELLE À SUPERVISER;}

\section{DOIT-ON S'INQUIÉTER?}

- achat de médicaments en vente libre, de vitamines, médecines alternatives, médicaments clandestins

(club d'achat);

- consommation de médicaments en investigation, d'urgence ou de recherche;

- consommation de substances récréatives, de médicaments empruntés à une connaissance;

- vérification de ce que le malade pense prendre et ce que l'on pense qu'il prend versus ce qu'il prend en réalité;

- identification des médicaments à risque d'être impliqués dans une interaction médicamenteuse:

- la dose du médicament est-elle faible ou élevée?

- la dose du médicament a-t-elle été modifiée récemment ou sera-t-elle modifiée?

- l'indice thérapeutique est-il étroit (c'est-à-dire le rapport entre la dose efficace et la dose toxique)?

- le niveau de médicament dans le sang est-il à l'état d'équilibre?

- le médicament sera-t-il administré pendant une longue période?

- y a-t-il une seule voie d'élimination pour ce médicament?

- le malade a-t-il des caractéristiques ou des conditions particulières pouvant modifier la réponse pharmacologique habituelle? (Anon, 2002)

Plusieurs malades ont recours aux médicaments en vente libre (sans ordonnance) de façon rémittente ou continue. Cette notion est importante autant lors d'association de substances que lors de retrait d'une entité médicamenteuse du régime contemporain (Patsalos et al., 2002).

L'utilisation des produits à base d'herbes aux États-Unis a augmenté autant que 380\% dans la dernière décennie (MacKichan et Ruthman, 2004). Plusieurs médicaments issus de la médecine moderne proviennent du monde végétal. Par exemple, la morphine est dérivée des graines de pavot (opium). Le millepertuis, le ginseng, le ginkgo, l'ail, le ephedra, le kava, la valériane, l'echinacée et la camomille sont des produits doués de vertus thérapeutiques mais qui peuvent à plusieurs égards juguler, entraver ou décupler l'activité d'entités médicamenteuses. (MacKichan et Ruthman, 2004). En 1999, aux ÉtatsUnis, au palmarès des produits botaniques les plus populaires figuraient le gingko, le millepertuis, le ginseng, l'ail et l'echinacée, chacun se partageant environ $10 \%$ du marché. L'emploi concomitant de produits de la botanique et de médicaments d'ordonnance peut résulter en échec thérapeutique, voire en catastrophe clinique (Huang et al., 2004).

Les médicaments les plus souvent utilisés en soins palliatifs incluent les opiacés, les anti-inflammatoires, les coxibs, les neuroleptiques, les antidépresseurs tricycliques, les inhibiteurs sélectifs de la recapture de la sérotonine, les neuroleptiques, les agents adjuvants, les laxatifs, les antiémétiques, les corticostéroïdes, les anticoagulants, les antibiotiques (Bernard et Bruera, 2000).

Les interactions médicamenteuses peuvent être étiquetées comme étant de nature pharmacocinétique ou pharmacodynamique (voir le tableau 1). La pharmacodynamique est caractérisée par la nature même de ce que le médicament fait à l'organisme. Les interactions qui touchent cette catégorie résultent d'une compétition pour le site récepteur (effecteur), des organes cibles ou des changements physiologiques et exprimant soit une synergie, soit un antagonisme (Davis et Homsi, 2001). Bien que les interactions pharmacocinétiques soient nombreuses et bien décrites, les interactions pharmacodynamiques documentées ne sont pas légion et habituellement déduites par défaut. Ces interactions peuvent être sommatives (additives ou synergiques) ou antagonistes au regard de la physiologie de l'organisme (Maurer et Bartowski, 1993).

Un exemple de ceci est l'adjonction d'un agent qui constipe ou ralentit la vidange de l'estomac alors qu'un malade reçoit un traitement par métoclopramide [Maxeran ${ }^{\circledR}$, Reglan ${ }^{\circledR}$ ] (un médicament qui active la vidange de l'estomac). Une interaction de type pharmacodynamique peut produire une toxicité additive; par exemple, le syndrome sérotoninergique qui peut découler de la combinaison d'un antidépresseur inhibiteur sélectif de la recapture de la sérotonine (médicament antidépresseur aussi employé pour la douleur, p. ex., paroxétine [Paxil $\left.{ }^{\circledR}\right]$ ) avec millepertuis (un produit naturel) ou avec le dextrométorphane (sirop DM) [la combinaison d'agents peut augmenter le taux de sérotonine dans le sang et donner lieu à un tel syndrome avec manifestations de différents niveaux de toxicité - voir le tableau 1]. 


\section{TABLEAU 1 - TYPES D'INTERACTIONS MÉDICAMENTEUSES}

1. Interactions pharmaceutiques. Incompatibilité physicochimique (visible ou invisible à l'œil $\mathrm{nu}$ ) se produisant entre deux médicaments et pouvant modifier l'intégrité de ceux-ci (formation de précipité, de floculation, de décoloration, de solution trouble). Ex. : médicament ajouté à un soluté ou médicaments (deux ou plus) ajoutés en seringues (médicaments en injection), solutions pour voie orale ou entités solides ou poudres ajoutées aux préparations de gavage, au lait ou aux différents jus.

2. Interactions pharmacodynamiques. Modification de la réponse pharmacologique globale (effet recherché, effet indésirable ou effet toxique) se produisant lorsque deux médicaments (ou plus) qui agissent sur un même système, un même transmetteur, un même modulateur ou un même récepteur sont associés. L'interaction peut être rapide et prévisible (ex.: effets indésirables similaires) ou imprévisible, rare voire fatale (ex. : syndrome sérotoninergique - décrit plus bas).

- Compétition pour le site d'action, le récepteur ou l'activation:

- morphine et autres opiacés similaires - naloxone $\left(\operatorname{Narcan}{ }^{\circledR}\right)$ [le naloxone antagonise l'effet des opiacés];

- Similitude ou antagonisme pour l'activité d'un système:

- deux benzodiazépines (p. ex., clonazépam [Rivotril ${ }^{\circledR}$ ] et oxazépam [Serax ${ }^{\circledR}$ ] ou lorazépam $\left[\right.$ Ativan $\left.^{\circledR}\right]$ ). Les deux sont prescrites alors qu'elles ont le même effet;

- un anti-inflammatoire (p. ex., ibuprofène[Motrin ${ }^{\circledR}$ ] ou naproxen [Naprosyn ${ }^{\circledR}$ ] et warfarine[Coumadin ${ }^{\circledR}$ ], un médicament pour éclaircir le sang; ils augmentent le risque de saignement;

- warfarine (Coumadin ${ }^{\circledR}$ ) et produits naturels (ginkgo bilboa, ail, griffe du diable, ginseng...); ils augmentent le risque de saignement;

- anti-inflammatoires et antihypertenseurs; ils réduisent l'effet des antihypertenseurs;

- morphine et autres opiacés - méthylphénidate $\left(\right.$ Ritalin $\left.{ }^{\circledR}\right)$; cela antagonise les effets de dépression respiratoire, les effets sédatifs de la morphine et des autres opiacés et les effets cognitifs; effets analgésiques potentiellement additifs;

- morphine et autres opiacés - chlorpromazine (Largactil $\left.{ }^{\circledR}\right)$, méthotriméprazine $\left(\right.$ Nozinan $\left.{ }^{\circledR}\right)$; cela entraîne une majoration de l'hypotension, de la sédation et des effets analgésiques;

- dextrométorphane (sirop DM pour la toux) et ISRS - dextrométorphane et mépéridine $\left(\right.$ Demerol $^{\circledR}$ ); aussi carbamazépine -ISRS (syndrome sérotoninergique hyperexcitabilité, hyperthermie, rigidité, diaphorèse, hypertension, tachycardie, tremblements, convulsions, cyanose, hypotension, coma).

(Voir Anon, 2002; Maurer et Bartowski, 1993.)

3. Interactions pharmacocinétiques. Modification des propriétés pharmacocinétiques du ou des médicaments impliqués lorsque deux ou plusieurs médicaments sont associés ou encore sous l'influence d'une substance cessée. La modification peut être immédiate ou retardée et entraîner un changement de la réponse thérapeutique globale.

Les médicaments peuvent affecter la motilité gastro-intestinale et, conséquemment, l'absorption d'autres médicaments administrés de façon concomitante. Les prokinétiques augmentent la vidange de l'estomac et la motilité du tube digestif supérieur. Ces effets peuvent favoriser une augmentation de la vitesse d'absorption initiale de médicaments par voie orale mais réduisent la biodisponibilité totale de ces agents.

Les opiacés ainsi que les médicaments doués de propriétés anticholinergiques peuvent retarder la vidange de l'estomac et influencer ce qui arrive aux autres médicaments. Même si ceci n'a pas de répercussions cliniques de façon générale, pris individuellement les conséquences pourraient avoir des retombées palpables surtout si le médicament touché possède un indice thérapeutique faible et que son absorption est pauvre sans l'impact d'une interaction actualisée (Greiff et Rowbotham, 1994).

Les interactions pharmacocinétiques sont des événements non désirés; elles surviennent lorsqu'un médicament protagoniste affecte d'une façon quelconque l'absorption, la distribution, le métabolisme ou l'excrétion. Ce type d'interaction implique des changements de mécanique du corps pour le métabolisme d'un médicament lors de l'ajout d'un autre médicament ou d'une substance. La plupart des interactions se manifestent à l'étape de la biotransformation hépatique (Davis et Homsi, 2001). (Les interactions d'absorption par l'organisme et d'excrétion par le rein ne seront pas abordées dans le présent texte.)

De nombreuses interactions sont décrites dans la documentation scientifique mais plusieurs relèvent de rapports de cas empiriques ou de données in vitro. Ces rapports indiquent des résultats qui ne sont pas toujours faciles à extrapoler (par exemple, les doses ou les indications mentionnées ne sont pas les mêmes que pour le cas index en présence), sont souvent faits pour une prise de médicaments à court terme et n'indiquent pas de facto la conduite à tenir. La documentation pour l'effet d'associations médicamenteuses comportant des interactions multiples - à contresens et inversement - est peu manifeste, voire inexistante.

Les médicaments qui possèdent un faible indice thérapeutique (rapport entre la dose efficace et la dose toxique) et qui sont susceptibles de subir ou d'induire une interaction préjudiciable demandent une attention spécifique (p. ex., phénytoïne [Dilantin ${ }^{\circledR}$ ], digoxin $\left[\right.$ Lanoxin $\left.^{\circledR}\right]$, méthadone $\left[\right.$ Metadol $\left.^{\circledR}\right]$, théophylline [Théodur ${ }^{\circledR}$ ], warfarine [Coumadin ${ }^{\circledR}$ ]). (Voir Anon, 2002.)

\section{DISTRIBUTION \\ (transport du médicament dans le sang et dans les tissus)}

Liaison aux protéines: les protéines agissent comme des capteurs de molécules et leur travail consiste à transporter ces dernières vers le foie en vue d'une transformation et d'une élimination. Le déplacement d'un médicament de ses sites de fixation aux protéines plasmatiques par un autre médicament augmente la fraction libre (active et produisant l'action pharmacologique) du premier médicament (Anon, 2002; Patsalos et al., 2002). Cela implique une plus grande quantité de médicaments sous forme libre pouvant accéder aux récepteurs pour exercer une action pharmacologique mais également une plus grande quantité de médicament disponible pour l'élimination. Conséquemment, les répercussions cliniques sont le plus souvent négligeables mais certains médicaments comme l'acide valproïque $\left(\right.$ Epival $\left.{ }^{\circledR}\right)$ [médicament utilisé pour l'analgésie, les troubles de l'humeur ou l'épilepsie], la phénytoïne $\left(\right.$ Dilantin $\left.^{\circledR}\right)$ [médicament utilisé pour la douleur et l'épilepsie], les salicylates, la warfarine $\left(\right.$ Coumadin $^{\circledR}$ ) sont des molécules à surveiller (Anon, 2002).

Le potentiel pour une interaction significative par déplacement de liaison aux protéines tient si les conditions suivantes sont réunies:

- Le médicament est fortement lié aux protéines;

- Le médicament possède un petit volume de distribution;

- Le médicament démontre un faible indice thérapeutique (c'est-à-dire que le ratio entre la dose efficace et la dose toxique est peu élevé);

- La vitesse d'élimination est lente. 
sournoisement

l'ombre est venue

d'un chemin inconnu

Une interaction de cette nature en soins palliatifs implique la co-administration d'acide valproïque $\left(\right.$ Epival $\left.^{\circledR}\right)$ et de phénytoïne (Dilantin ${ }^{\circledR}$ ) [déplacement du second par le premier]. De surcroît, l'acide valproïque est un inhibiteur du métabolisme de la phénytoïne (2C9). La warfarine (Coumadin ${ }^{\circledR}$ ), un anticoagulant (médicament pour éclaircir le sang, par exemple, pour le traitement d'une thrombophlébite ou d'une embolie pulmonaire), est également une substance qui présente un potentiel pour actualiser une telle interaction lors d'association médicamenteuse (Bernard et Bruera, 2000).

\section{MÉTABOLISME}

\section{(processus en vue de faciliter I'élimination des médicaments)}

L'estomac, les reins, les poumons et le cerveau ont la capacité de métaboliser les différents xénobiotiques mais l'intestin et le foie représentent les principaux sites connus actuellement. Le médicament qui subit la transformation est communément appelé substrat (Anon, 2002)).

La désintoxication des xénobiotiques inclut les toxines, carcinogènes et médicaments et devient une tâche centrale des systèmes enzymatiques du métabolisme de l'organisme.

Le cytochrome P450 (CYP 450) consiste en un groupe d'enzymes localisés dans les hépatocytes (cellules du foie) et le long de la surface muqueuse du tube digestif. La fonction de ces enzymes consiste à veiller à la transformation endogène des stéroïdes, des hormones, des prostaglandines et des lipides; ce système comporte une deuxième mission, la transformation des xénobiotiques (Davis et Homsi, 2001).

Parce que le système du CYP450 catalyse ultimement la première étape de la transformation des médicaments, cette fonction est appelée métabolisme de phase I. La phase I précède souvent diverses étapes de conjugaison, aussi appelées phase II. Le métabolisme de phase II est généré par différents systèmes enzymatiques, le plus important étant celui de l'UDGP (uridyl diphosphate glucuronosyltransférases). Les isoformes de cette enzyme conjuguent les composés en dérivés glucuroniques, faisant de ces substrats des entités plus hydrosolubles et donc disponibles pour l'excrétion par l'urine ou

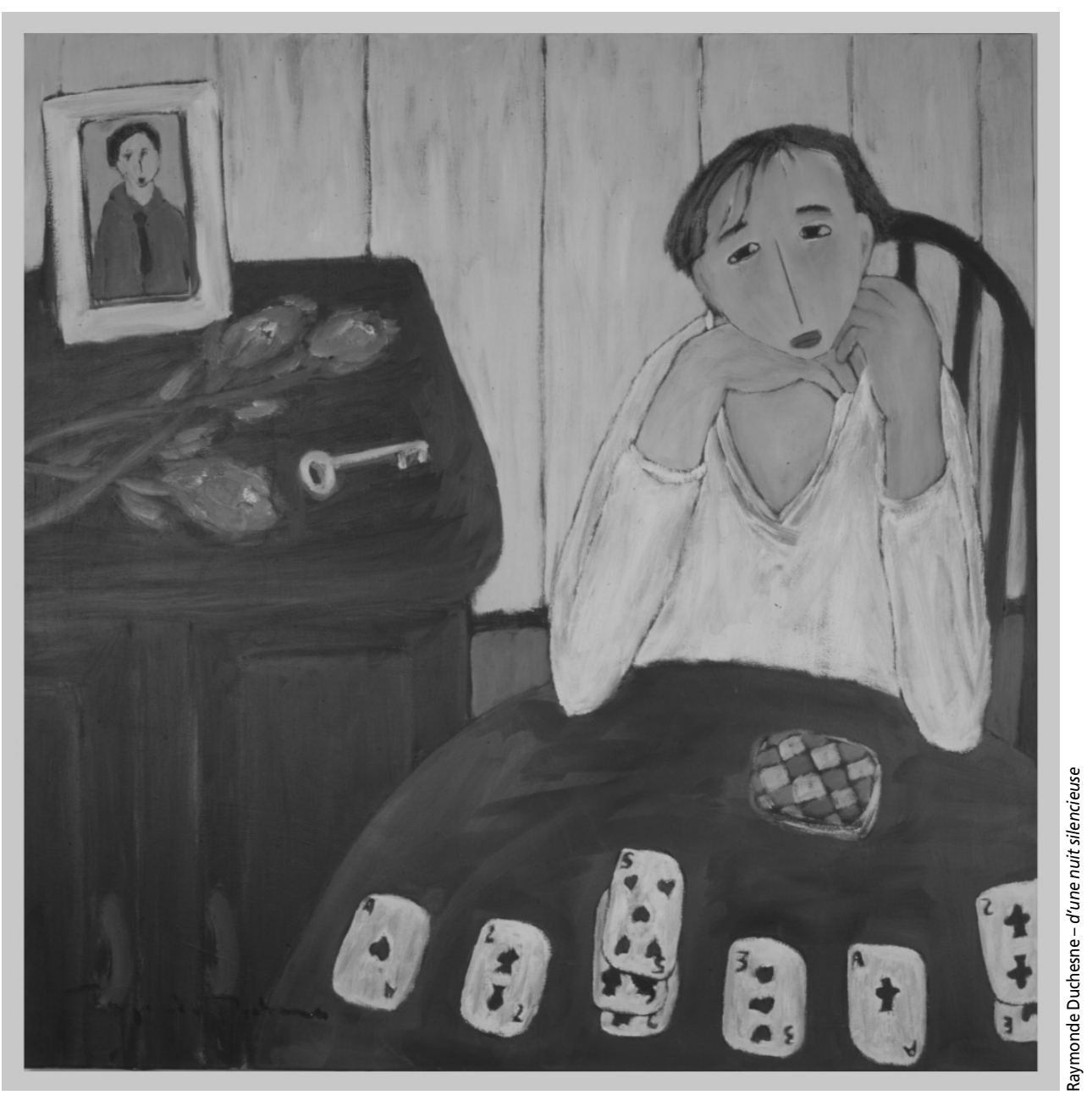

par la bile. D'autres systèmes enzymatiques importants pour cette deuxième phase sont les glutathione-S-transférases, les sulfotransférases et les acétyltransférases (qui ne seront pas traitées dans le présent texte).

Les recherches les plus récentes ont démontré que les étapes de transport devraient être ajoutées à nos modèles connus de défense des xénobiotiques. Des systèmes de pompes permettant d'expulser les agresseurs permettent de réduire le fardeau local cellulaire de composés toxiques conférant ainsi à l'individu une protection cellulaire contre les effets toxiques d'une agression. Les protéines de transport (comme la p-glycoprotéine) sont principalement exprimées sur le pôle le plus externe de la membrane des cellules épithéliales, comme les entérocytes (cellules du tube digestif), qui sont exposés aux xénobiotiques exogènes. Au sein de ces cellules, le même transporteur travaille pour réduire l'entrée de substances d'une part et d'autre part pour éliminer les produits biotransformés. Cette dernière étape est appelée phase III du métabolisme, indiquant une intrication des phases métaboliques. La première étape, qui consiste en une extrusion de l'organisme avant l'entrée dans la cellule, peut être identifiée comme étape de phase 0 du métabolisme (Dietrich et al., 2003).

Tous les médicaments qui sont exclus de l'organisme par le biais d'un de ces systèmes d'épuration sont susceptibles de subir ou d'engendrer une interaction médicamenteuse lorsqu'ils entrent en dynamique avec d'autres substances.

Chez l'humain, le système enzymatique du cytochrome $\mathrm{P} 450$ est reconnu pour métaboliser un nombre considérable d'entités médicamenteuses et autres xénobiotiques. Le système cytochrome $\mathrm{P} 450$ consiste en une superfamille douée de capacité métabolique oxydative pour de multiples substances. Il s'agit d'un des systèmes enzymatiques les plus connus pour le métabolisme des médicaments (Ma et al., 2002). Ce système existe sous de multiples formes ou isoenzymes (aussi appelés isoformes), chacun affichant une distribution variable dans les différents tissus. La base de la nomenclature se réfère aux séquences génétiques des isoenzymes; on retrouve des familles représentées par un chiffre arabe (similitude dans la séquence des protéines qui constituent les unités d'une même famille d'enzymes), le CYP450-1, CYP450-2, CYP450-3 et CYP450-4. Les familles se subdivisent en sous-familles désignées par une lettre 


\section{LE CYP450-2D6}

De façon préférentielle, le CYP450-2D6 métabolise la classe des antidépresseurs tricycliques, les inhibiteurs sélectifs de la recapture de la sérotonine (nouvelle lignée des antidépresseurs depuis les années 1990), des neuroleptiques (halopéridol $\left[\right.$ Haldol $\left.^{\circledR}\right]$, rispéridone [Risperdal ${ }^{\circledR}$ ], aussi appelés tranquillisants majeurs et employés pour leurs effets analgésique, antiémétique, anxiolytique ou sédatif), de la codéine (un opiacé), de l'hydrocodone (Hydodan ${ }^{\circledR}$, un opiacé), de l'oxycodone (Supeudol ${ }^{\circledR}$, Percocet $^{\circledR}, \operatorname{OxyContin}^{\circledR}$, un opiacé), des $\beta$-bloqueurs (métoprolol [Lopresor ${ }^{\circledR}$, propranolol [Inderal $\left.{ }^{\circledR}\right]$ ) et des antiarythmiques (propafénone $\left[\right.$ Rytnmol $\left.^{\circledR}\right]$, flécaïnide [Tambocor $\left.{ }^{\circledR}\right]$ ) utilisés pour leurs propriétés antiarythmique ou analgésique (Davis et Homsi, 2001).

L'activité du CYP450-2D6 peut, entre autres, être entravée par l'action de la quinine (que l'on peut retrouver comme médicament et comme agent de conservation dans la boisson Water Tonic), la quinidine, la méthadone, la diphenhydramine

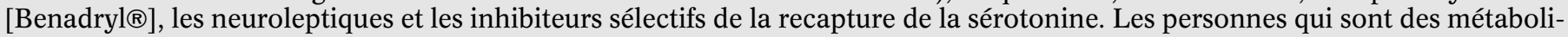
sateurs lents peuvent être vulnérables et éprouver plus facilement des effets indésirables aux doses habituelles des médicaments métabolisés par le 2D6 mais généralement elles ne sont pas sensibles aux interactions puisque leur activité métabolique est déjà limitée par le polymorphisme qu'ils expriment (métabolisateur lent par déficience relative en 2D6). Les métabolisateurs extensifs eux sont particulièrement à risque d'interaction puisque l'influence d'un autre médicament pouvant inhiber le métabolisme (extensif) du premier aura un retentissement sans conteste (Davis et Homsi, 2001).

Le dextrométorphane (sirop DM pour la toux, cette substance possède des qualités antalgiques) est facilement retrouvé sur les tablettes des comptoirs du pharmacien et accessible sans le besoin d'une ordonnance du médecin. Chez les métabolisateurs lents, les niveaux plasmatiques de dextrométorphane sont de 15 à 25 fois plus élevés que chez les personnes dont le métabolisme est normal (rapide). Le dextrométorphane augmente la libération de sérotonine du cerveau. Ce médicament est un substrat du CYP450-2D6 et est susceptible de subir l'assaut des médicaments qui nuisent à l'activité de l'isoenzyme. Les répercussions anticipées sont des hallucinations potentielles et un syndrome sérotoninergique (Davis et Homsi, 2001; Bernard et Bruera, 2000).

\section{Les psychotropes (aussi appelés neuroleptiques)}

Plusieurs psychotropes sont métabolisés par le CYP450-2D6; la chlorpromazine (Largactil ${ }^{\circledR}$ ), l'halopéridol, la rispéridone, l'olanzapine $\left(\right.$ Zyprexa $\left.^{\circledR}\right)$, la méthotriméprazine $\left(\right.$ Nozinan $\left.^{\circledR}\right)$ sont transformés en partie ou totalement par cette voie. L'halopéridol, les doses élevées de méthotriméprazine et les doses standards de chlorpromazine sont aussi inhibitrices du 2D6. Ils sont des inhibiteurs plus puissants que les antidépresseurs tricycliques à ce chapitre. La co-administration de quinine ou de quinidine et d'un neuroleptique peut mener à une majoration de l'effet de ce dernier pouvant être préjudiciable en termes d'effets extrapyramidaux (raideur, mouvements anormaux et stéréotypés du corps). Voir Davis et Homsi, 2001.

\section{Les antidépresseurs tricycliques \\ (amitriptyline $\left[\right.$ Elavi $\left.\left.\right|^{\circledR}\right]$, désipramine $\left[\right.$ Norpramin $\left.{ }^{\circledR}\right]$, nortriptyline $\left[\right.$ Aventy $\left.\left.\right|^{\circledR}\right]$, clomipramine $\left[\right.$ Anafranil $\left.{ }^{\circledR}\right]$, imipramine [Surmonti ${ }^{\circledR}$ ], utilisés pour leur effet analgésique ou antidépresseur)}

La plupart d'entre eux sont des substrats du CYP450-2D6, mais ils n'inhibent ni n'induisent ce dernier. L'interaction entre les tricycliques et les inhibiteurs sélectifs de la recapture de la sérotonine (ISRS) se produit principalement par l'inhibition du CYP par les ISRS. Cette interaction qui peut parfois nuire si elle n'est pas planifiée est souvent mise à contribution dans le plan thérapeutique prévu (Davis et Homsi, 2001).

\section{Les inhibiteurs sélectifs de la recapture de la sérotonine (ISRS) (paroxétine $\left[\right.$ Paxil $\left.^{\circledR}\right]$, fluoxétine $\left[\right.$ Prozac $\left.^{\circledR}\right]$, sertraline [Zoloft $\left.{ }^{\circledR}\right]$, fluvoxamine [Luvox $\left.{ }^{\circledR}\right]$, venlafaxine [Effexor $\left.{ }^{\circledR}\right]$, citalopram [Celexa $\left.{ }^{\circledR}\right]$ )}

Le métabolisme de ces médicaments est accompli par la voie de différentes familles et sous-familles du CYP.

La fluoxétine est transformée en son métabolite actif par la voie de l'isoforme 2D6; la paroxétine est inactivée par le 2D6; la fluvoxamine est transformée par le CYP450-1A2 et le 2D6; la sertraline par le 3A4; le citalopram par le 2C19 et le 3A4.

La puissance inhibitrice des ISRS en regard de l'impact sur le CYP450-2D6 est en ordre décroissant: paroxétine > fluoxétine et norfluoxétine (métabolite actif) $>$ sertraline $>$ fluvoxamine $>$ venlafaxine. Chacune de ces molécules, et dans un ordre de puissance différent de celui susmentionné, peut également inhiber l'activité d'autres isoenzymes et ainsi potentiellement augmenter les effets d'un autre médicament administré et transformé par cette voie.

Par exemple, la fluvoxamine, un inhibiteur sélectif de la recapture de la sérotonine, est un puissant inhibiteur du CYP4501A2 et du 2C19 et un inhibiteur plus modeste du 3A4, 2C9 et 2D6. Chez l'humain, la fluvoxamine augmente considérablement les concentrations plasmatiques de substrats tels la ropivacaïne (Naropin ${ }^{\circledR}$, un anesthésique local utilisé par l'anesthésiste pour soulager la douleur), la caféine, la théophylline (Théodur ${ }^{\circledR}$, un médicament qui ouvre les bronches chez les asthmatiques), la clozapine (Clozaril ${ }^{\circledR}$, un médicament qui calme l'agitation). Voir Granfors et al., 2004.

\section{Venlafaxine (Effexor $\left.{ }^{\circledR}\right)$}

La conversion en un métabolite actif se fait par le biais du CYP450-2D6 et à un degré moindre par le 2C19. Il faut être prudent lors de la co-administration avec les antidépresseurs tricycliques, les $\beta$-bloqueurs et certains agents neuroleptiques.

\section{La mirtazapine (Remeron ${ }^{\circledR}$, un médicament pour les troubles de l'humeur et du sommeil)}

Cette molécule est un substrat de plusieurs cytochromes: le CYP1A2, le CYP3A4, le CYP2C9 et le CYP2D6; la voie principalement empruntée pour le métabolisme est le CYP3A4 et à un niveau moindre le CYP2D6. La paroxétine et la fluoxétine peuvent retarder la clairance de la mirtazapine lors de co-administration d'une ou de l'autre entité. La carbamazépine peut augmenter l'élimination de la mirtazapine en augmentant l'activité du 3A4. La mirtazapine possède elle-même de faibles propriétés inhibitrices sur l'activité du CYP2D6, 1A2 et 3A4 (Davis et Homsi, 2001). 
(ex.: CYP1A ou CYP2C), enfin un gène particulier encode 1 'isoenzyme et est identifié par un second chiffre arabe (ex.: CYP1A2 ou CYP2C9); (identification du gène qui fait la besogne pour éliminer un certain nombre de médicaments [substrats]).

En plus de son occupation au sein des hépatocytes, la distribution extra-hépatique du CYP450 (notamment le CYP450-3A4) est extensive; on le retrouve notamment au niveau de l'intestin, des canaux biliaires, $\mathrm{du}$ poumon, du rein, de la peau, du cerveau. La paroi intestinale, hormis son système de transporteurs qui encourage l'élimination des médicaments (comme susmentionné), est très riche en isoenzymes du cytochrome P450 (Davis et Homsi, 2001; Turgeon et Michaud, 2002).

Le substrat d'une enzyme est une molécule qui comporte une affinité pour les protéines de cette enzyme. Un médicament est un substrat d'une isoenzyme du cytochrome P450 si cette isoenzyme particulière est capable de transformer cette molécule par une réaction chimique en métabolite.

Une molécule est l'inducteur d'une isoenzyme du cytochrome P450 si elle est capable d'augmenter l'activité de cette dernière. Généralement l'inducteur augmente la synthèse (donc la quantité) d'enzymes présentes, ce qui en augmente l'activité. Les interactions d'induction sont plus longues à s'exprimer comparativement aux interactions d'inhibition. Linduction requiert la synthèse de nouveaux enzymes et peut nécessiter plusieurs jours, voire des semaines avant la répercussion clinique factuelle (Patsalos et al., 2002).

Comme l'induction est un processus progressif, le temps requis pour l'induction et donc pour que l'interaction devienne probante et manifeste est tributaire de certains paramètres: le temps nécessaire pour que l'agent inducteur soit à l'équilibre dans le sang (ce qui peut prendre de quelques heures à plusieurs jours) et la vitesse de synthèse de nouveaux enzymes. La valeur de l'induction est également proportionnelle à la dose de l'inducteur.

Le temps requis pour terminer l'interaction au retrait du médicament responsable dépend de la vitesse de la dégradation des enzymes et du temps requis pour éliminer le médicament inducteur (Anderson, 1998).

Les inhibiteurs sont des substances qui possèdent une affinité pour une isoenzyme du cytochrome P450 mais qui entrainent une baisse d'activité de cette dernière lorsqu'elles y sont fixées (Turgeon et Michaud, 2002).

L'inhibition hépatique se produit habituellement parce qu'un médicament entre en compétition avec un autre médicament pour un même site de transformation; ceci résulte en une diminution du métabolisme d'un des deux agents puisqu'il perd sa place

\section{LE CYP450-3A4}

- Un certain nombre de benzodiazépines, d'antidépresseurs tricycliques et les anticonvulsivants classiques (phénytoïne [Dilantin $\left.{ }^{\circledR}\right]$, carbamazépine [Tegretol $\left.{ }^{\circledR}\right]$ ) ont des propriétés d'inducteurs du CYP450-3A4. Des inhibiteurs puissants à ce niveau sont, entre autres, l'érythromycine et le kétoconazole.

- Les substrats incluent certaines benzodiazépines (midazolam [Versed ${ }^{\circledR}$ ], alprazolam $\left[\operatorname{Xanax}^{\circledR}\right]$, triazolam [Halcion $\left.{ }^{\circledR}\right]$ ), les anticonvulsivants classiques (carbamazépine), les opiacés (méthadone, fentanyl [en partie]), les antifongiques (fluconazole [Diflucan ${ }^{\circledR}$ ], itraconazole [Sporanox ${ }^{\circledR}$ ], terbinafine [Lamisil ${ }^{\circledR}$ ], kétoconazole [Nizoral $\left.{ }^{\circledR}\right]$ ), la quinine, la quinidine, l'amiodarone [Cordarone $\left.{ }^{\circledR}\right]$, les antihistaminiques de nouvelle génération (loratadine [Claritin $\left.{ }^{\circledR}\right]$ ), les antiinfectieux (azithromycine [Zithromax ${ }^{\circledR}$ ], érythromycine, clarithromycine $\left[\right.$ Biaxin $\left.^{\circledR}\right]$ ), les bloqueurs du canal calcique (diltiazem [Cardizem ${ }^{\circledR}$ ], vérapamil $\left[\right.$ Isoptin $\left.^{\circledR}\right]$ ), les corticostéroïdes (prednisone, dexaméthasone [Decadron $\left.{ }^{\circledR}\right], \ldots$ ), certains inhibiteurs sélectifs de la recapture de la sérotonine. Plusieurs de ces médicaments, en plus d'être métabolisés par cette voie, sont dotés de pouvoir inhibiteur de cette même voie de transformation (ex.: fluconazole, itraconazole, kétoconazole, diltiazem, vérapamil, érythromycine, clarithromycine). Voir Davis et Homsi, 2001.

sur le site de transformation. Sur le plan clinique, ceci est associé à une augmentation des taux plasmatiques du médicament qui subit l'interaction et, en conséquence, à une augmentation de l'effet pharmacologique (ce peut être un bienfait ou un mal). Le début de l'interaction se manifeste généralement rapidement, souvent à l'intérieur d'une période de 24 heures suivant l'ajout de l'inhibiteur (comparativement aux interactions d'induction) mais le temps pour la répercussion maximale dépendra du temps que prendront les deux médicaments (le médicament qui cause l'interaction et celui qui la subit) pour atteindre un équilibre dans le sang (Anderson, 1998).

Certaines isoenzymes démontrent un polymorphisme génétique; ceci signifie que différentes personnes peuvent exprimer des anomalies de certains isoenzymes de leur système enzymatique, ce qui donne lieu à des modifications dans leur capacité (augmentée, diminuée ou absente) à transformer des médicaments par rapport à la population normale (Davis et Homsi, 2001).

On identifie les métabolisateurs rapides, ce qui correspond au profil d'une majorité de personnes (75 à 85\% de la population), des métabolisateurs intermédiaires (10 à $15 \%$ ), des métabolisateurs lents (5 à 10\%) et des métabolisateurs très rapides (1 à 10\%). Pour compliquer un peu plus les choses, on observe que le polymorphisme peut s'exprimer différemment non seulement à l'intérieur d'une race mais qu'il existe des disparités entre les ethnies (Meyer, 2000).

L'isoenzyme le mieux étudié du CYP450 est le 2D6. (Pour plus de détails, voir l'encadré sur le CYP450-2D6.) La plupart des individus sont des métabolisateurs rapides, mais une proportion d'individus sont des métabolisateurs très rapides (extensifs) alors que d'autres sont des métabolisateurs lents.
Une partie de la population caucasienne (5 à 10\%) exprime un métabolisme ralenti ou absent par rapport au CYP450-2D6. Ceci donne lieu à une réponse pharmacologique différente de celle attendue pour une population en général lors de l'instauration d'un traitement par un médicament empruntant cette voie.

Ces individus s'exposent à une réponse augmentée à un médicament lorsque l'activité de ce dernier est reliée à la substance mère, c'est-à-dire le produit avant transformation. À l'inverse, si c'est le produit de transformation (le métabolite actif) qui exerce un effet pharmacologique, ces personnes sont exposées à une diminution de l'effet escompté, voire à un échec thérapeutique.

Par exemple, la codéine nécessite une conversion en morphine par le biais du CYP450-2D6 pour étayer son action pharmacologique. Si l'activation du médicament ne peut pas se produire ou est réprimée, la répercussion se traduira par une diminution, voire une absence d'efficacité.

Les patients qui sont des métabolisateurs lents ne peuvent obtenir d'effet analgésique avec la codéine puisqu'ils ne peuvent transformer la codéine en morphine (Davis et Homsi, 2001; Hajela, 2004).

La portion normalement transformée en morphine est probablement responsable de l'activité analgésique obtenue chez la plupart des gens qui métabolisent adéquatement. Les patients qui ne répondent pas à la codéine et qui se voient prescrire une rotation pour un autre opiacé (alors que la dose de codéine avait été majorée sans succès) sont susceptibles de subir les effets délétères d'un tel changement d'agent si l'on se fie au ratio analgésique lors de la conversion sans tenir compte de la déficience enzymatique possible. Ces individus, le plus souvent, lorsqu'ils seront exposés à deux médicaments passant par la voie du 


\section{LES ANTICONVULSIVANTS}

- La phénytoïne: elle augmente le métabolisme de médicaments qui sont transformés par le CYP450-2C9 et 2C19, le CYP450-3A3/4 et l'UDGT. Le temps pour l'induction maximale et à l'inverse lors de l'arrêt de la phénytoïne est en moyenne de une à deux semaines (les répercussions ne sont donc pas immédiates). (Anderson, 1998.)

La phénytoïne est elle-même métabolisée par la voie des CYP notamment le CYP450-2C9 et à un moindre niveau le 2C19 et peut donc être entravée dans son élimination par d'autres molécules pouvant inhiber (fluconazole [Diflucan ${ }^{\circledR}$ ] un médicament pour les infections à champignons; amiodarone, acide valproïque, oméprazole [Losec ${ }^{\circledR}$ ] un médicament pour protéger l'estomac...) ou induire (carbamazépine) ces isoenzymes (Anderson, 1998.)

- Le phénobarbital: il augmente le métabolisme de médicaments qui sont transformés par le CYP450-2C9 et 2C19, le CYP450-3A3/4 et l'UDGT. Le temps pour l'induction maximale et à l'inverse lors de l'arrêt du phénobarbital est en moyenne de deux à trois semaines (début après une semaine). (Anderson, 1998.)

- La carbamazépine (Tegretol ${ }^{\circledR}$ ): il augmente le métabolisme de médicaments qui sont transformés par le CYP450-2C9 et 2C19, le CYP450-3A3/4 et l'UDGT. Le temps pour le début de l'induction est de une semaine et pour l'induction maximale peut être de plusieurs semaines et à l'inverse lors de l'arrêt de la carbamazépine est en moyenne de une à trois semaines. La caractéristique particulière de la carbamazépine est qu'elle peut auto-induire son propre métabolisme. La carbamazépine, entre autres, peut aussi induire le métabolisme des antidépresseurs tricycliques (Anderson, 1998).

La carbamazépine est métabolisée par la voie du CYP450-3A4 et, à un degré moindre, par le CYP2C8 et 1A2. Les interactions avec la carbamazépine sont fort complexes puisqu'elle comporte un métabolite actif. Notons celles plus connues avec la fluoxétine, la phénytoïne, l'acide valproïque, l'érythromycine, la clarithromycine, de certains bloqueurs du canal calcique - médicaments pour le cœur (inhibition de la transformation de la carbamazépine et/ou de son métabolite actif). (Anderson, 1998.)

- L'acide valproïque (ou valproate) : il est reconnu comme étant un inhibiteur de la transformation des médicaments substrats des CYP450-2C, de l'UDGT et d'autres systèmes enzymatiques. Ce médicament entrave notamment l'élimination de la phénytoïne, du phénobarbital, de la lamotrigine (Lamictal ${ }^{\circledR}$ ), du lorazépam (Ativan®). (Anderson, 1998.)

L'acide valproïque (ou valproate) est lui-même substrat de l'UDGT et, à un niveau moindre, du CYP450-2C (Anderson, 1998).

- La lamotrigine $\left(\right.$ Lamictal $\left.{ }^{\circledR}\right)$ : elle induit son propre métabolisme et cause une diminution légère des taux de l'acide valproïque (Epival ${ }^{\circledR}$ ) lorsque les deux médicaments sont combinés.

Les concentrations de lamotrigine sont diminuées par l'adjonction de carbamazépine, phénytoïne ou de phénobarbital. L'acide valproïque (ou valproate) donne lieu à une augmentation significative des taux sériques de lamotrigine; la combinaison des deux agents a donné lieu à l'apparition de manifestations sur la peau ou de réaction d'hypersensibilité parfois grave. L'édification de ligne de conduite stricte a été recommandée et réalisée pour une majoration parcimonieuse de la dose de lamotrigine quand l'adjonction est incontournable (Anderson, 1998).

- Le topiramate $\left(\right.$ Topamax $\left.^{\circledR}\right)$ : il inhibe le métabolisme de médicaments substrats du CYP450-2C19. Le topiramate peut lui-même subir les contrecoups d'une association médicamenteuse notamment lors de combinaison avec la phénytoïne ou le phénobarbital (baisse des concentrations de topiramate de 40 à 50\%).(Anderson, 1998.)

CYP450-2D6 pour leur transformation, ne subiront pas d'effet notoire d'une telle interaction. Comme leur métabolisme est déjà lent, l'effet d'un médicament inhibiteur aura peu de répercussions (Meyer, 2000).

Autrement, les métabolisateurs rapides en regard du CYP450-2D6 et soumis aux effets de plus d'un médicament dont l'un peut inhiber la transformation de l'autre par la voie de ce CYP pourront vivre les effets drastiques d'une telle association. On parle alors de métabolisateurs rapides devenus métabolisateurs lents sous l'influence d'un inhibiteur enzymatique. Ce type de polymorphisme relié à des facteurs environnementaux plutôt qu'à l'hérédité est réversible au retrait du médicament ou du facteur incriminé (Meyer, 2000). Un polymorphisme a également été identifié dernièrement pour le CYP450-2C9, le 2C19 et le 1A2 (Bernard et Bruera, 2000).

Plusieurs médicaments peuvent inhiber de multiples isoenzymes, alors que d'autres ne peuvent inhiber qu'un seul isoenzyme. Le métabolite d'un médicament plutôt que la substance mère elle-même (c'est-à-dire le produit de transformation d'un médicament) peut devenir inhibiteur ou inducteur de l'activité enzymatique; il peut arriver que la substance mère (c'est-à-dire le produit à l'origine) et le métabolite soient doués de propriétés inhibitrices ou inductrices. Le degré d'interaction peut également être influencé par l'affinité pour le cytochrome (site enzymatique). Les inhibiteurs et inducteurs de faible puissance ont peu de répercussion sur le métabolisme global (sauf dans certaines circonstances). La combinaison du polymorphisme et des interactions médicamenteuses mène parfois à des disparités notoires dans la clairance d'un médicament en particulier entre les sous-populations d'individus. De plus, une substance peut être à la fois le substrat pour une enzyme (c'est-à-dire le produit à être transformé par l'enzyme) et un inhibiteur ou un inducteur pour cette même enzyme. Par exemple, la paroxétine (Paxil ${ }^{\circledR}$ ) est à la fois un substrat et un inhibiteur de l'isoenzyme 2D6 du CYP450 (elle peut auto-inhiber sa propre transformation). La méthadone et la carbamazépine (Tegretol ${ }^{\circledR}$ ) auto-induisent l'isoenzyme 3A4 du CYP450, accélérant leur propre élimination à partir du foie. Un médicament peut être métabolisé par plusieurs isoenzymes et alors l'inhibition d'un des isoenzymes aura peu de portée clinique; autrement, le médicament et son métabolite pourraient partager la même efficacité et dans ce cas l'inhibition n'aura vraisemblablement pas de signification contextuelle (p. ex., la rispéridone [Risperdal $\left.{ }^{\circledR}\right]$ ). (Davis et Homsi, 2001.)

L'isomère 2D6 (pour plus de détails, voir l'encadré sur le CYP450-2D6) ne compte que pour 2 à $5 \%$ de la capacité du système mais ceci représente $25 \%$ des médicaments existants et souvent prescrits en soins palliatifs (Davis et Homsi, 2001).

L'isomère $3 \mathrm{~A} 4$ compte pour 40 à $60 \%$ de la capacité enzymatique totale du CYP450 et $56 \%$ des médicaments actuels sont métabolisés par les mono-oxygénases (Davis et Homsi, 2001).

Le CYP450-3A4 est responsable d'une grande partie des interactions médicamenteuses connues (pour plus de détails, voir l'encadré sur le CYP450-3A4). Contrairement au 2D6, il ne semble pas y avoir de polymorphisme manifeste pour cet isoenzyme qui peut être inhibé ou induit. L'activité de l'isoenzyme 3A varie de 10 à 40 fois entre les individus (Davis et Homsi, 2001). 
L'activité du CYP450-3A4 intestinal réduit la fraction d'un médicament pouvant être absorbé par le tube digestif. En plus de cette isoenzyme, la p-glycoprotéine contribue également à l'extrusion d'un médicament vers la lumière intestinale freinant ainsi son accès vers l'organisme et par là même son action thérapeutique. Cette protéine travaille de concert avec le CYP450 intestinal pour épurer l'organisme des xénobiotiques avant même qu'ils franchissent d'autres barrières. On retrouve la p-glycoprotéine au niveau du rein, du cerveau et des canaux biliaires. On peut donc comprendre qu'un médicament possédant un pouvoir inhibiteur à la fois sur le CYP450-3A4 intestinal, hépatique et sur la p-glycoprotéine pourra de façon très marquée modifier la pharmacocinétique d'un agent métabolisé par ces voies (Davis et Homsi, 2001).

\section{LES ANTICONVULSIVANTS (utilisés pour la prise en charge de certains syndromes douloureux ou les accès convulsifs)}

La plupart des anticonvulsivants connus sont éliminés par métabolisme hépatique et passent par la voie des cytochromes et d'un autre système enzymatique appelé UDGT (uridine diphosphate glucuronosyltransférase). La phénytoïne, le phénobarbital, la carbamazépine peuvent stimuler le travail de l'UDGT et de plusieurs CYP et ainsi accélérer l'élimination d'autres médicaments administrés et excrétés par cette voie. L'acide valproïque (ou le valproate) est un inhibiteur de l'UDGT et du CYP450-2C. Le topiramate $\left(\right.$ Topamax $^{\circledR}$ ) inhibe l'activité du CYP4502C19. (Pour plus de détails, voir l'encadré sur les anticonvulsivants). La gabapentine (Neurontin $^{\circledR}$ ) n'exerce aucune influence sur les différents systèmes enzymatiques responsables de la transformation des médicaments en vue de leur élimination (Anderson, 1998). La gabapentine créant moins d'artifices a supplanté les anciens anticonvulsivants aux fins d'analgésie et est devenu un premier choix dans cette classe de médicaments.

\section{LES ANALGÉSIQUES NON OPIACÉS}

L'acétaminophène $\left(\right.$ Tylenol $^{\circledR}$, Atasol ${ }^{\circledR}$ ) est détoxiqué par le foie par le CYP450-2E1, 1A2 et 3A4. La toxicité hépatique de ce médicament est bien connue d'où l'importance de ne jamais dépasser la dose quotidienne recommandée et d'éviter la surconsommation d'alcool avec ce produit. En effet l'alcool et l'acétaminophène empruntent la même voie de transformation par le CYP laissant place à des niveaux plasmatiques persistants d'acétaminophène. L'alcool est aussi un inducteur de l'isoenzyme accélérant ainsi la transformation de l'acétaminophène en son métabolite intermédiaire hépatotoxique (Barkin et al., 2001).
Les anti-inflammatoires non stéroïdiens (AINs) peuvent exercer des interactions médicamenteuses de nature pharmacodynamique; ils occasionnent une rétention d'eau et de sel (sodium) dans l'organisme et ainsi peuvent déstabiliser un traitement de l'hypertension ou de l'insuffisance cardiaque. (Barkin et al., 2001)

Plusieurs anti-inflammatoires (naproxen [Naprosyn ${ }^{\circledR}$ ], diclofénac [Voltaren ${ }^{\circledR}$ ], ibuprofène [Motrin ${ }^{\circledR}$ ]) et les coxibs (célécoxib [Celebrex ${ }^{\circledR}$ ], valdécoxib [Bextra $\left.{ }^{\circledR}\right]$ ) sont d'excellents substrats du CYP450 - notamment le 2C9, 2C18, 2C19 pour leur transformation métabolique. Ils sont donc sujets à subir et engendrer des interactions d'ordre pharmacocinétique également (Barkin et al., 2001; Ma et al., 2002).

\section{LES RELAXANTS MUSCULAIRES (employés pour leur activité relaxante ou analgésique pour lutter notamment contre la spasticité)}

La cyclobenzaprine (Flexeril®) subit une transformation par plusieurs voies enzymatiques; le CYP450-1A2, 2D6 et 3A4 sont responsables de son devenir. Le médicament peut donc subir les affres d'une association médicamenteuse, mais les conséquences peuvent être épargnées par les relais possibles entre les circuits d'élimination (Barkin et al., 2001) .

Le cytochrome P450-1A2 pourrait être l'isoforme impliqué dans le métabolisme de la tizanidine (Zanaflex ${ }^{\circledR}$ ).

Les antidépresseurs et les relaxants sont passablement employés et parfois en association dans les syndromes douloureux chroniques. L'association de fluvoxamine et de tizanidine peut rapidement donner préséance à des symptômes s'apparentant à une inhibition de la transformation de la tizanidine (hypotension grave, diminution de la fréquence cardiaque, somnolence, étourdissements, diminution de la performance psychomotrice). (Granfors et al., 2004.)

\section{LES OPIACÉS PUISSANTS}

Les principaux métabolites de la morphine sont des conjugués de l'acide glucuronique, à savoir le morphine-6glucuronide (M6G), qui a une activité analgésique, et le morphine-3-glucuronide (M3G), qui n'a pas d'activité analgésique. Ces deux métabolites sont principalement excrétés dans l'urine et peuvent donc s'accumuler en insuffisance rénale. L'hydromorphone est aussi métabolisé par glucuronidation. Ses métabolites, dont l'hydromorphone3-glucuronide (H3G), ne semblent pas avoir d'activité analgésique significative.

La codéine et l'oxycodone sont métabolisés par la sous-famille du CYP450-
2D6, alors que le fentanyl et la méthadone sont métabolisés par la sous-famille CYP450-3A4.

Contrairement à la codéine, l'oxycodone affiche une activité analgésique sous sa forme initiale. Par conséquent, les métabolisateurs lents peuvent être soulagés à l'aide de petites quantités d'oxycodone, car sa dégradation par la sous-famille CYP4502D6 est lente (Hajela, 2004).

Comme le fentanyl et la méthadone sont métabolisés par le système CYP4503A4, ils sont susceptibles d'être affectés par induction ou inhibition du CYP450 en raison de l'administration concomitante d'autres médicaments. L'induction enzymatique peut survenir avec l'administration chronique puisque certains médicaments stimulent leur propre métabolisme ainsi que d'autres agents métabolisés par la même voie (Hajela, 2004).

Les opiacés interagissent avec d'autres médicaments par de multiples mécanismes, résultant en effets pharmacologiques qui dépendent de l'action pharmacodynamique étudiée, des agents concomitants employés et de la voie d'administration. La transformation de certains opiacés est dépendante de la capacité du foie à métaboliser les produits (fentanyl, alfentanil, sufentanil, méthadone). Les antibiotiques sont souvent prescrits en concomitance: l'érythromycine est reconnue comme pouvant augmenter les effets des opiacés (alfentanil) alors que la rifampicine à tout coup en jugule les actions. La carbamazépine, la phénytoïne et les barbituriques peuvent mousser le métabolisme des opiacés qui dépendent de la capacité enzymatique du foie (système des CYP450 ; Maurer et Bartkowski, 1993).

D'autres interactions, pharmacodynamiques cette fois, avec les opiacés impliquent les benzodiazépines, les antidépresseurs tricycliques et les phénothiazines (sédation additive).

\section{LES FACTEURS ENVIRONNEMENTAUX CONNUS}

Le tabagisme possède un effet bien connu qui a été quantifié lors d'études expérimentales dans lesquelles on a observé que l'activité enzymatique chez les fumeurs réguliers (20 cigarettes ou plus par jour) était 1,72 fois plus élevée que chez les non-fumeurs.

Après l'arrêt du tabagisme, on a observé une diminution statistiquement significative de l'activité du CYP450-1A2 (baisse de $12,3 \%$, de $20,1 \%$, de $28,2 \%$ et de $36 \%$ respectivement aux jours 1 à 4 et à l'équilibre; Faber et Fuhr, 2004). Ce phénomène peut avoir des retombées percutantes sur le métabolisme des médicaments empruntant cette voie de transformation (ex.: ciprofloxacin $\left[\right.$ Cipro $\left.^{\circledR}\right]$ un antibiotique, théophylline [Théodur $\left.{ }^{\circledR}\right]$, olanzapine $\left[\right.$ Zyprexa $\left.^{\circledR}\right]$ ). 
La cuisson au charbon et les aliments crucifères (choux, brocoli, choux-fleur, choux de Bruxelles) ingérés en quantité suffisante sont de bons inducteurs enzymatique du CYP450-1A2.

Le jus de pamplemousse administré pour une période de 5 jours $(200 \mathrm{~mL}$ par jour de façon régulière) a mené à une augmentation modeste de la biodisponibilité (quantité de médicament disponible pour l'effet thérapeutique) et à une diminution équivalente de la clairance de la méthadone. Un tel effet est probablement attribuable au fait que le jus de pamplemousse (à cette dose de $200 \mathrm{~mL}$ ) augmente la biodisponibilité des médicaments en freinant de façon sélective la régulation de l'expression du CYP4503A4 intestinal; l'effet est donc plus marqué sur les médicaments présentant une faible biodisponibilité (ex.: la nifédipine [Adalat ${ }^{\circledR}$ ], la simvastatine $\left[\right.$ Zocor $\left.\left.^{\circledR}\right]\right)$. (Benmebarek et al., 2004.)

L'ingestion de grandes quantités de jus de pamplemousse (6 à 8 verres par jour) peut conduire à l'inhibition du CYP-450 3A4 hépatique cette fois-ci. Le jus de pamplemousse contribue à la réponse pharmacologique exagérée remarquée avec certains bloqueurs du canal calcique, le diltiazem $\left(\right.$ Cardizem $^{\circledR}$ ), le vérapamil (Isoptin ${ }^{\circledR}$ ) [hypotension, étourdissements, syncope], certaines statines, la simvastatine $\left(\right.$ Zocor $\left.^{\circledR}\right]$ ) [myalgie, rhabdomyolyse] et autres agents thérapeutiques. Il est possible que le jus de pamplemousse et d'autres boissons fruitées puissent affecter d'autres transporteurs intestinaux (ex.: p-glycoprotéine). (Huang et al., 2004.)

Une situation où une toxicité peut se produire est lorsqu'un patient se voit administrer une dose plus haute que la dose usuelle d'un médicament susceptible et que cette personne boit pour la première fois $\mathrm{du}$ jus de pamplemousse. Ceci peut se produire si le médecin augmente la dose d'un médicament pour obtenir un effet pharmacologique. Cette dose, chez un patient possédant un haut contenu en CYP450-3A4 intestinal, peut être titrée à un équivalent de dose relativement élevée pour obtenir l'effet escompté. Un freinage soudain de l'activité intestinale enzymatique, comme cela peut se produire lors de la consommation de jus de pamplemousse, peut résulter en toxicité médicamenteuse.

Une autre situation peut survenir lorsqu'une personne souffrant de maladie hépatique sévère dépend presque essentiellement de son CYP-450 intestinal pour le métabolisme de certains médicaments. De telles personnes peuvent être exposées à des niveaux toxiques de substances administrées à doses conservatrices; une inhibition du CYP intestinal chez ces patients peut avoir de répercussions notoires sans conteste.
Finalement, les individus prédisposés à une susceptibilité pour une toxicité à certains médicaments, même à dose conventionnelle, seront plus vulnérables et sensibles à une inhibition de la transformation métabolique (Huang et al., 2004).

\section{LES MÉDICAMENTS EN VENTE LIBRE ET LES PRODUITS NATURELS}

L'emploi de ces produits est monnaie courante; l'automédication est souvent le recours de personnes qui veulent soulager certains symptômes non apaisés par les médicaments d'ordonnance ou tout simplement parce qu'ils ne peuvent communiquer avec leur médecin.

Les produits botaniques sont particuliers en ceci que le plus souvent ils consistent en un mélange de substances complexes comprenant un ou plusieurs ingrédients actifs connus ou inconnus.

Le mythe voulant que les produits soient naturels et sans danger est en partie élucidé. L'expérience nous a démontré que malheureusement cela était faux et depuis, on s'acharne à mieux comprendre et analyser ces produits où la notion de pureté du principe actif est quasi illusoire (sauf exception). Les mêmes notions et principes d'interactions médicamenteuses s'appliquent ici (Scott et Elmer, 2002).

La camomille est un inhibiteur du CYP 450-3A4; elle possède des effets additifs avec les benzodiazépines et autres agents qui dépriment le système nerveux central (Scott et Elmer, 2002).

L'adjonction d'echinacée et d'alprazolam $\left[\mathrm{Xanax}^{\circledR}\right]$, une benzodiazépine pour le sommeil et les troubles de l'humeur, peut augmenter les niveaux sériques et donc les effets de ce dernier. Théoriquement, une personne qui entame un traitement pour un rhume par echinacée et qui consomme régulièrement du jus de pamplemousse peut voir croître de façon substantielle son risque d'interaction médicamenteuse avec un autre médicament (Scott et Elmer, 2002).

L'echinacée peut inhiber le CYP4503A4 intestinal, inhiber le CYP450-2C9 et 1A2 hépatiques et induire le CYP450-3A4 hépatique (Huang et al., 2004 ; Gorski et al., 2004).

Une interaction grave a été rapportée entre le ginkgo et la trazodone (Desyrel ${ }^{\circledR}$ ), un médicament pour les troubles du sommeil; l'association a donné lieu à une augmentation du métabolite actif de la trazodone. Le mécanisme présumé pouvant expliquer cette interaction est une induction du CYP 3A4 ou une interaction pharmacodynamique sur certains récepteurs (Scott et Elmer, 2002).

L'ipriflavone présente des qualités d'inhibiteur métabolique du CYP450-1A2 pouvant donc influencer le devenir de médicaments comme la théophylline, la caféine, la clozapine, l'olanzapine, la cyclobenzaprine, l'halopéridol, etc. Certaines données de laboratoire nous indiquent que l'ipriflavone peut entraver l'activité du CYP4502C9; des médicaments importants et qui sont substrats de ce CYP sont la phénytoïne, les AINs, le célécoxib, la warfarine (Scott et Elmer, 2002).

La mélatonine souvent employée par nos compatriotes américains et européens pour contrer le décalage horaire ou l'insomnie est vulnérable aux interactions. Métabolisée par la voie du CYP450-1A2 et $2 \mathrm{C} 9$, elle est sujette à une persévérance de son activité lorsque sa transformation est inhibée par un médicament compétiteur (ex.: fluvoxamine, halopéridol, diazépam). (Scott et Elmer, 2002.)

Des publications récentes rapportent qu'une co-administration de millepertuis a donné lieu à une diminution des taux plasmatiques d'indinavir, de cyclosporine et de digoxin menant à un constat d'échec thérapeutique grave (Huang et al., 2004).

L'administration de millepertuis régulièrement et pour une période dépassant 14 jours donne place à une induction du CYP450-3A4 intestinal et de la p-glycoprotéine (Huang et al., 2004).

\section{INSUFFISANCE HÉPATIQUE ET INSUFFISANCE RÉNALE}

Les études histologiques ont démontré que l'insuffisance hépatique chronique évolutive pouvait avoir une répercussion sur le métabolisme des médicaments. Conséquemment, comme la cirrhose progresse, le flot sanguin hépatique efficace diminue, et la clairance des médicaments qui dépendent du flot hépatique devient de plus en plus limitée par la capacité métabolique; ces agents deviennent plus sensibles à l'action des inducteurs et inhibiteurs enzymatiques (Orlando et al., 2004).

Le résultat d'études examinant les niveaux des protéines du CYP chez les patients insuffisants hépatiques révèle des effets différents, certaines étant profondément diminuées, d'autres moins et d'autres pas. Les résultats les plus consistants en regard des changements sur le système enzymatique du foie chez les patients souffrant de cirrhose touchent le CYP450-1A2; on a observé des diminutions importantes, et cela, dans plusieurs études (Orlando et al., 2004).

L'enzyme du système P-450 le plus abondant, le CYP-3A4, est connu pour être impliqué dans la transformation de plus de 200 médicaments. Dans les modèles animaux d'insuffisance rénale, autant la fonction hépatique que le contenu en enzymes du CYP sont affectés; toutefois les évidences chez l'humain sont manquantes. L'évaluation des capacités métaboliques 
des personnes à un stade avancé de l'insuffisance rénale est cruciale et pivot; les individus affectés sont exposés à une panoplie d'entités médicamenteuses les rendant vulnérables à moult effets indésirables et interactions médicamenteuses(Dowling et al., 2003).

Une association entre la maladie rénale et un pouvoir réduit dans le métabolisme hépatique a été observée dans les modèles animaux; une corrélation significative a été relevée entre les indices de la fonction rénale (BUN et créatinine sérique) et la fonction du foie (CYP450) dans ces modèles. Ceci laisse supposer que la fonction hépatique (et le métabolisme) puisse être altérée en présence d'insuffisance rénale et supporte l'hypothèse que l'accumulation de toxines urémiques puissent inhiber le métabolisme hépatique soit en réduisant la capacité des enzymes (contenu CYP) ou en inhibant la capture des médicaments par les hépatocytes (Dowling et al., 2003).

L'évaluation des effets d'un dysfonctionnement du rein, malgré une hémodialyse adéquate, sur le système CYP-450 a d'importantes répercussions pharmacothérapeutiques (Dowling et al., 2003).

Des évidences indirectes avec l'emploi de certains médicaments chez les individus touchés par une insuffisance rénale supportent l'hypothèse d'une capacité de transformation amoindrie. La clairance non rénale pourrait être diminuée de 30 à $50 \%$ en présence d'insuffisance rénale et chez les malades en hémodialyse (Dowling et al., 2003).

Ces dernières années, une approche plus rationnelle est adoptée en regard des interactions médicamenteuses; notre compréhension du système enzymatique CYP-450 responsable du devenir métabolique de plusieurs xénobiotiques nous permet d'affiner notre jugement clinique et d'anticiper certains dangers. Une appréciation des principes fondamentaux qui gouvernent les voies de transformation permet de mieux régir le plan thérapeutique afin d'obtenir une réponse progressive et planifiée.

Notre connaissance du rôle du polymorphisme génétique dans le métabolisme des médicaments qui empruntent les voies enzymatiques pour permettre de déterminer comment un individu répond à certaines combinaisons d'agents s'améliorera avec le temps. Éventuellement, la pharmacogénétique pourra mener à des tests de dépistage permettant au prescripteur de sélectionner la combinaison médicamenteuse la plus appropriée pour un individu donné selon son empreinte génétique.

Armé de cette habileté d'anticipation pour un potentiel d'interaction médicamenteuse respectant le médicament, le profil biochimique, la condition et les comorbidités du malade, le médecin sera apte à prendre une décision plus rationnelle quant à une polypharmacie, assurant ainsi un effet thérapeutique maximal pour une toxicité minimale. Malgré tout ce savoir, les interactions demeurent singulièrement imprévisibles; pour une partie des individus, elles seront manifestes alors que, pour d'autres, elles passeront inaperçues.

Il faut remettre en lumière que les interactions anticipées peuvent ne pas s'actualiser chez tous les patients qui se voient prescrire une combinaison médicamenteuse indiquée et que ces associations peuvent être mises à profit pour le malade.

\section{Bibliographie}

ANDERSON, G.D. (1998). "A mechanistic approach to antiepileptic drug interaction », Ann. Pharmacother., vol. 32, p. 554-563.

ANON (2002). «Interactions médicamenteuses », dans Regroupement de pharmaciens en établissement de santé ayant un intérêt pour les soins palliatifs, Guide pratique des soins palliatifs: gestion de la douleur et autres symptômes, $3^{\mathrm{e}}$ éd., Montréal, A.P.E.S., p. 211-235

BENMEBAREK, M., C. DEVAUD, M. GEX-FABRY, K. POWEL GOLAY, C. BROGLI, P. BAUMANN et al. (2004). «Effects of grapefruit juice on the pharmacokinetics of the enantiomers of methadone», Clin. Pharmacol. Ther., vol. 76, $\mathrm{n}^{\mathrm{0}}$ 1, p. 55-63.

BERGK, V., C. GASSE, D. ROTHENBACHER, M. LOEW, H. BRENNER et W.E. HAEFELI (2004). "Pharmacoepidemiology and drug utilisation. Drug interactions in primary care: Impact of a new algorithm on risk determination», Clin. Pharmacol. Ther., vol. 76, $\mathrm{n}^{\circ} 1$, p. 85-96.

BERNARD, S.A. et E. BRUERA (2000). "Drug interaction in palliative care», J. Clin. Oncol., vol. 18, no 8, p. 1780-1799.

DAVIS, M.P. et J. HOMSI (2001). "The importance of cytochrome P450 monooxygenase CYP2D6 in palliative medicine", Support Care Cancer, vol. 9, p. 442-451.

DIETRICH, C.G, A. GEIER et R.P.J. OUDE ELFERINK (2003). "ABC of oral bioavailability: Transporters as gatekeepers in the gut», Gut, vol. 52, p. 1788-1795.

DOWLING, T.C., A.E. BRIGLIA, J.C. FINK, D.S. HANES, P.D. LIGHT, L. STACKIEWICZ et al. (2003). "Characterisation of hepatic cytochrome P4503A activity in patients with end-stage renal disease», Clin. Pharmacol. Ther., vol. 73, no 5, p. 427-434.

FABER, M.S. et U. FUHR (2004). "Time response of cytochrome P450 1A2 activity on cessation of heavy smoking », Clin. Pharmacol. Ther., vol.76, $\mathrm{n}^{\circ} 2$, p. 178-184.

GORSKI, J.C., S.M. HUANG, A. PINTO, M.A. HAMMAN, J.K. HILLIGOSS, N.A. ZAHEER et al. (2004). "The effect of (Echinacea purpura root) on cytochrome P450 activity in vivo ", Clin. Pharmacol. Ther., vol. $75, \mathrm{n}^{\circ} 1$, p. 89-100.
GRANFORS, M.T., J.T. BACKMAN, M. NEUVONEN, J. AHONEN et P.J. NEUVONEN (2004). "Fluvoxamine drastically increases concentrations and effects of tizanidine: A potentially hasardous interaction», Clin. Pharmacol. Ther., vol. 75, $\mathrm{n}^{\mathrm{o}}$ 4, p. 331-341.

GREIFF, J.M.C. et D. ROWBOTHAM (1994). «Pharmacokinetic drug interactions with gastrointestinal motility modifying agents », Clin. Pharmacokinet, vol. 27, $\mathrm{n}^{\circ} 6$, p. 447-461.

HAJELA, R. (2004). «Traitement opiacé chronique: problèmes de métabolisme, d'interactions médicamenteuses et de toxicomanie», La gestion de la douleur, vol. 4, n ${ }^{\circ}$ 4, p. 3-7.

HUANG, S.M., S.D. HALL, P. WATKINS, L.A. LOVE, C. SERABJIT-SINGH, J.M. BETZ et al. (2004). "Drug interactions with herbal products and grapefruit juice: A conference report», Clin. Pharmacol. Ther., vol. $75, \mathrm{n}^{\circ} 1$, p. 1-12.

KIN, R.L. et D. BARKIN (2001). «Pharmacologic management of acute and chronic pain: Focus on drug interactions and patientspecific pharmacotherapeutic selection", South Med. J., vol. 94, no 8, p. 756-770.

MA, M.K., M.H. WOO et H.L. MCLEOD (2002). "Genetic basis of drug metabolism », Am. J. Health-Syst. Pharm., vol. 59, $\mathrm{n}^{\circ}$ 21, p. 2061-2069.

MACKICHAN,C. et C. RUTHMAN (2004). "Herbal product use and perioperative patients », AORN Journal, vol. 79, $\mathrm{n}^{\circ}$ 5, p. 948-959.

MAURER, P.M. et R.R. BARTKOWSKI (1993). "Drug interaction of clinical signifiance with opioid analgesics », Drug Safety, vol. $8, \mathrm{n}^{\circ} 1$, p. 30-48.

MEYER, U.A. (2000). «Pharmacogenetics and adverse drug reactions ", Lancet, vol. 356, $\mathrm{n}^{\circ} 11$, p. $1667-1671$.

ORLANDO, R., P. PICCOLI, S.D. DEMARTIN, R. PADRINI, M. FLOREANI et P. PALATINI (2004). "Pharmacokinetics and drug disposition. Cytochrome P450 1A2 is a major determinant of lidocaine metabolism in vivo : Effects of liver function", Clin. Pharmacol. Ther., vol. 75, $\mathrm{n}^{\circ} 1$, p. 80-88.

PATSALOS, P.N., W. FRÖSCHER, F. PISANI et C.M. VAN RIJN (2002). "The importance of drug interactions in epilepsy therapy», Epilepsia, vol. 43, n 4, p. 365-385.

PERRI, D., S. ITO, V. ROWSELLL et N.H. SHEAR (2003). "The kidney. The body playground for drugs: An overview of renal drug handling with selected clinical correlates", Can. J. Clin. Pharmacol., vol. $10, \mathrm{n}^{\mathrm{o}} 1$, p. 17-23.

SCOTT, G.N. et G.W. ELMER (2002). "Update on natural product-drug interactions", Am. J. Health-Syst. Pharm., vol. 59 , n ${ }^{\circ} 4$, p. 339-347.

TURGEON, J. et V. MICHAUD (2002). "Le cytochrome P450 et les interactions médicamenteuses", L'actualité médicale (supplément), juin, p. 3-6. 